\title{
Wissenschaft, Fortbildung und Nachwuchsförderung in der Inneren Medizin
}

Es ist ein gesundheitspolitisches Thema, welches längst die Medien und die Politik erreicht hat: In Teilbereichen der klinischen und der ambulanten Versorgung, vor allem in strukturschwachen, ländlichen Regionen, droht ein Ärztemangel. Zugleich verlassen viele Ärzte Deutschland, um im Ausland ihre Facharztausbildung zu absolvieren und dort ihre berufliche Zukunft zu suchen. In den verantwortlichen Gremien und Ministerien existieren durchaus vielfältige Vorstellungen, wie man junge Ärzte für Tätigkeiten in der Klinik und in Praxen gewinnen kann; diese reichen von einer Lockerung des Numerus clausus über neue Arbeitszeitmodelle bis hin zu finanziellen Anreizen für Landarztpraxen. In dieser Diskussion wird indes häufig der Aspekt vernachlässigt, dass in noch stärkerem Maße ein wissenschaftlicher Nachwuchsmangel in der Medizin droht. Es ist zunehmend schwieriger geworden, junge Mediziner neben ihrer Facharztausbildung für wissenschaftliche Fragestellungen zu begeistern.

Die Deutsche Gesellschaft für Innere Medizin (DGIM) hat diese Problematik gerade auch für die klinisch-internistische Forschung in den vergangenen Jahren erkannt und auf ihre Agenda gesetzt. Mit ihrer Kommission „Wissenschaft, Nachwuchsförderung“ wurden Konzepte erarbeitet und Positionspapiere zu aktuellen Themen veröffentlicht. Der wissenschaftliche Nachwuchs benötigt frühzeitig im Rahmen seiner universitären Tätigkeit die Möglichkeit zur selbstständigen Forschung, die in die Weiterbildung integriert ist. Unser Positionspapier „Anrechenbarkeit von Forschungstätigkeit während der Weiterbildungszeit" ist daher von unveränderter Aktualität. In Analogie zu den Weiterbildungscurricula verschiedener europäischer Fachgesellschaften der Inneren Medizin müssen auch in Deutschland (erneut) Initiativen zur Anerkennung krankheitsorientierter Forschungstätigkeit auf die Weiterbildungszeit ergriffen werden. Die Förderung des weiblichen wissenschaftlichen Nachwuchses (s. auch das entsprechende Positionspapier) wurde von der DGIM während des zurückliegenden Jahres als ein besonderes Anliegen gesehen. Frauen stellen in der Humanmedizin inzwischen $61 \%$ der Studienanfänger und über 50\% der Promovierenden, sind aber im akademischen Mittelbau sowie im Anteil der Professorinnen und Chefärztinnen deutlich unterrepräsentiert.
Viele Medizinerinnen erleben das Umfeld für eine wissenschaftliche Karriere als unattraktiv oder als hinderlich in ihrer Bestrebung, Beruf und Familie zu vereinbaren. Die DGIM hält es für dringend erforderlich, durch gezielte und verstärkte Anstrengungen auf allen Ebenen (Universitäten, Universitätsklinika, außerhochschulischen Forschungseinrichtungen, Krankenhäusern) und Bereichen wie der Deutschen Forschungsgemeinschaft (DFG) und wissenschaftlichen Fachgesellschaften dazu beizutragen, das zur Verfügung stehende Potenzial von Studienabsolventinnen mehr als bisher für die Wissenschaft und für die qualitativ hochwertige Besetzung von Führungspositionen zu erschließen. Die Universitätsklinika sollten ihre Möglichkeiten ausbauen, durch gezielte Personalentwicklungsmaßnahmen eine intensive Begleitung in der Phase des Übergangs von der Promotion zur Habilitation zu gewährleisten. Als Orientierung könnten hier die „Forschungsorientierten Gleichstellungsstandards“ der DFG aus dem Jahr 2008 dienen. Von der DGIM werden zudem spezielle Mentoring- bzw. Coaching-Seminare für Nachwuchs-Wissenschaftlerinnen in der Inneren Medizin angeboten.

Fortbildung in der Inneren Medizin und ihren Schwerpunkten, up-to-date, lebendig und mit praktischer Relevanz für alle internistisch interessierten Kollegen, stellt für eine wissenschaftliche Fachgesellschaft wie die DGIM eine kontinuierliche Aufgabe dar. Mit dieser Ausgabe „Klinischer Fortschritt“ 6/2011 der Deutschen Medizinischen Wochenschrift schließen wir den ersten Jahrgang unserer DGIM-Gesellschaftsausgaben ab. Wir die Schwerpunkt-Herausgeber und Vorstandsmitglieder der DGIM - hoffen, dass wir mit dem aktuellen Überblick über hochrangige Publikationen und neue Leitlinien aus dem gesamten Spektrum der Inneren Medizin dem Anspruch der wissenschaftlich fundierten kontinuierlichen Fortbildung entsprochen und die Erwartungen der Leserinnen und Leser erfüllt haben. Der Jahrgang 2012 ist bereits konzipiert, in der konkreten Ausgestaltung sind wir für Anregungen, Kritik und Verbesserungsvorschläge dankbar!

Ihre

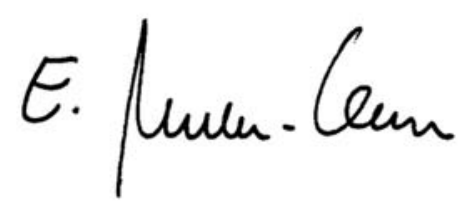

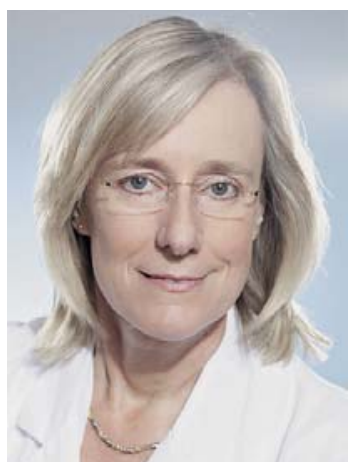

E. Märker-Hermann

Schlüsselwörter

Gesundheitspolitik

Ärztemangel

Nachwuchsförderung

Keywords

Dealth policy

shortage of physicians

promotion of young

physicians

Institut

Klinik Innere Medizin IV

(Rheumatologie,

Klinische Immunologie, Nephrologie), HSK Dr. Horst Schmidt Klinik GmbH

Bibliografie

DOI 10.1055/s-0031-1297264

Dtsch Med Wochenschr 2011;

136: 2453 - (c) Georg Thieme

Verlag KG Stuttgart · New York. ISSN 0012-0472

Korrespondenz

Prof. Dr. med.

Elisabeth Märker-Hermann

Klinik Innere Medizin IV (Rheumatologie, Klinische Immunologie, Nephrologie) HSK Dr. Horst Schmidt Klinik $\mathrm{GmbH}$

Ludwig-Erhard-Str. 100

65199 Wiesbaden

Tel. 0611/439777

Fax 0611/439779

eMail Elisabeth.Maerker-Her-

mann@HSK-Wiesbaden.de 